\title{
Resource Allocation for Efficient IOT Application in Fog Computing
}

\author{
Shubham Verma \\ Department of Computer Science and Engineering, \\ Rajkiya Engineering College, Kannauj, India. \\ Corresponding author: sverma705478@gmail.com \\ Amit Gupta \\ Department of Computer Science and Engineering, \\ Dr. A.P.J. Abdul Kalam Technical University, Lucknow, India. \\ E-mail: amitknp38@gmail.com \\ Sushil Kumar \\ Department of Information Technology, \\ Ajay Kumar Garg Engineering College, Ghaziabad, India. \\ E-mail: sushil0402k5@gmail.com \\ Vivek Srivastava \\ Department of Computer science and Engineering, \\ Rajkiya Engineering College, Kannauj, India. \\ E-mail: viveksrivastavakash@gmail.com

\section{Bipin Kumar Tripathi} \\ Department of Computer Science and Engineering, \\ Harcourt Butler Technical University, Kanpur, India. \\ E-mail: abkt.iitk@gmail.com
}

(Received December 14, 2019; Accepted July 15, 2020)

\begin{abstract}
When it comes across problems in creating Internet of Things (IOT) architecture, the major problem that arises is an automatic stipulation of resources. At the same time in today's era, it is very important to integrate this problem with better Quality of Services (QoS) because of which the cloud computing is taking a shift. As being well acquainted that in fog computing, network's bandwidth is limited, therefore it becomes quite important to build a joint architecture with resource allocation problem giving it a better quality of services with enhanced efficiency and low latency communication. Priority of QoS is determined by Systematic Ladder Process (SLP) and decision parameter evaluation by RECK algorithm. In this paper, there will be a design of a better framework for IOT resource allocation scheme with better efficiency and better QoS. The paper too highlights the comparison of the previous works of the resource allocation algorithms and schemes with RECK algorithm.
\end{abstract}

Keywords- IOT, QoS, Decision parameters, RECK algorithm.

\section{Introduction}

Since certain years, Internet of Things (IOT) has drawn consideration towards the field which incorporates industry and individuals in scholastics and it has profited the human life in an effective manner. Information taken by means of the sensors being keen in nature is typically conferred towards cloud centres of information and the application being normally being executed with the assistance of processors in these centres. 
International Journal of Mathematical, Engineering and Management Sciences

Vol. 5, No. 6, 1312-1323, 2020

https://doi.org/10.33889/IJMEMS.2020.5.6.097

On the opposite side of the coin, on the off chance that we see that amount of information created from the sensor and the cell phone that exponentially ascending at a more prominent rate and winding up huge and there are various powers being applied in the regions that incorporate correspondence and those of calculations incorporates into the system network inside different nature of administrations i.e. QoS essentials. Fog computing at that point said to be the arrangement given to expand the cloud computing element to the neighbourhood systems (Peng et al., 2016). After having the stars of the assets that are accessible towards the edge nodes and the converging of fog processing \& IOT systems had digressed the perspectives of the individuals in the diverse application domains. The fog calculation, numerous nodes being at the edge could contribute towards sharing of registering, storing and correspondence assets to numerical undertakings locally without interfacing with the cloud computing focus with the front haul likes (Ding et al., 2017; Islam et al., 2017; Shirvanimoghaddam et al., 2017; Song et al., 2017; Kiani and Ansari, 2018).

In this paper, we will assess the enhancement in asset assignment issue in fog figuring based IOT systems to fulfil the calculation and inactivity prerequisites of the IOT applications, with the intention to diminish the vitality utilization of the nearby calculation in the battery controlled IOT gadgets. Fog computing tackles of the issues of cloud computing, the enormous information stacked to the IOT gadgets to Fog nodes that requires the correspondence assets to fulfil both the transmission capacity and idleness necessities. Along these lines, both calculation and correspondence assets should be combined and booked to improve the exhibition of the framework. In the present period, IOT has expansive advancement in different fields, for example, keen home, well-being observing, natural and rural applications (Qiu et al., 2018; Rahman et al., 2019; Zhang et al., 2018). For dealing with a tremendous measure of information produced by IOT gadgets, different philosophy is advanced to feature numerous issues about fog figuring in remote IOT frameworks. So as to explain the client affiliation and the asset designation issue for broadband IOT applications in fog calculation, a two-side coin coordinating process was detailed depending on the assurance of QoS prerequisites needed in (Abedin et al., 2019).

The creators in (Fan and Ansari, 2018) acquainted drone base stations with move the weighted traffic heap of large-scale base stations and planned calculations for improvement of automaton arrangement and client relationship in fog IOT frameworks. The distributed computing space is profoundly proficient in the calculation and capacity assets for the IOT frameworks, yet the regularly expanding measure of asset necessities of the IOT applications had prompted the expanded vitality utilization and the presentation is corrupted of the registering hubs because of information transmission and processing fog relocation; accordingly, how to perform IOT applications turns into a difficult issue (Li et al., 2010; Luo et al., 2018; Peng et al., 2017; Sarkar et al., 2015; Tian et al., 2014). Fog computing stretching out the figuring procedures to the edge of the system as opposed to playing out the IOT applications in the cloud stage. In the fog condition, the router is a potential physical server, which could arrangement asset for the fog administrations at the edge of the system (Wang et al., 2016; Yi et al., 2015). The routers could expand the exhibition of calculation and capacity and which could be the full usage as the computing nodes. With the huge information in today's time, there are different execution imperatives for the IOT applications, particularly for the ongoing applications; in this way, such applications pick the edge registering nodes as a need to have (Abedin et al., 2019; Rahman et al., 2019). In the fog calculation the clients could get to and use the calculation and capacity, organize assets, similar to the manner in which the clients utilize the cloud asset, and the virtualized innovation is to give the on-request assets (Bonomi et al., 2014). The IOT applications could be performed by the fog computing nodes and the physical assets utilized in the remote cloud server farms. The asset portion of the IOT 
applications should mull over for both the brought together and the disseminated figuring nodes and the asset scheduler and supervisor ought to pick the suitable processing node to have the fog administration consolidated in the IOT applications by means of the plan of asset assignment procedures. The creating fog viewed is viewed as a fundamental square of IOT giving an enormous number of highlights, for example, low inactivity, area explicit, wireless access characteristics not at all like cloud computing. To provide the same features of cloud computing, fog devices are a part of IOT for performing computations and storage capacity. But the fog computing and IOT have the same target of providing better QoS. As the amount of traffic is increasing in IOT, QoS requirement is too increasing day by day. There should be low latency communication, low bit error rate and error free communication. So, we create a systematic ladder process to bifurcate the complex quality of service management and prioritize the QoS. Previously, many resource allocation algorithms are imparted which needs to be improved as per the demanding technology is moving towards higher efficiency. One of such algorithms is given in the following paper named as deferred algorithm (DA) (Gu et al., 2015).

\subsection{Deferred Accepting Algorithm}

It states that the resource allocation must be in a fashion that at least one stable matching should exist for the available list of resources available. The algorithm goes as:

1) Start

2) Initialise the user specified resource list or the preference list.

3) Each of its user puts a proposal of its favourite resource requests to delete the rest of the resources available in the resource list.

4) Acceptance or Rejection Phase: Accepting its favourite and rejecting rest by deletion.

5) Check If the users are matched or not.

6) If matched go 8 to else 7

7) Check if the resource list is empty or not, if yes goto 8 else goto 3.

8) Terminate: Output is the stable matching between the user and its respective resources.

9) End

Section 1 deals with the introduction where we try to define the previous researches and work in this field. It also includes the former algorithms that are related to the proposed algorithm and later on it proved to be beneficial to compare our algorithm to be effective. The remaining sections of this paper are organized as follows: Section 2 defines the network model that includes all the related terminologies which are responsible for the defining the network model with the utilization of various formulations and functions that are using in the proposed algorithm. Further the section 3 includes the problem statement and deals with how can all the benefits could be integrated under this section. Section 4 includes the decision parameter which deals with how the decision is being evaluated and problem solution to the problem defined in the previous sections. Section 5 determine various decision parameters on the basis of proposed algorithm RECK. Section 6 includes the various simulations that deals with the comparisons of RECK algorithm with DA algorithm and Section 7 discusses conclusion and impact of research with future scope.

\section{Network Model}

Network model defines all the tactics and notions that used to define the network architecture and the objects needed to define it are: the fog devices denoted by P, sub channels denoted by A, formulation for fixed bandwidth, IOT devices denoted by C, services needed by F, QoS parameters by $\mathrm{N}$ and followed by the formula for the weight of QoS Parameter. The notions are explained as below: 
International Journal of Mathematical, Engineering and Management Sciences

Vol. 5, No. 6, 1312-1323, 2020

https://doi.org/10.33889/IJMEMS.2020.5.6.097

Fog devices $\mathrm{P}=\{1 \ldots \ldots \mathrm{p}\}$

Sub-channel $\mathrm{A}=\{1 \ldots \ldots \mathrm{a}\}$

$x_{c, p}^{a}=$ Fixed bandwidth

No of IOT devices $\mathrm{C}=\{1 \ldots \mathrm{c}\}$

Services $\mathrm{F}=\{1 \ldots \mathrm{f}\}$

QoS parameter $\mathrm{N}=\{1 \ldots \mathrm{n}\}$

Weight of the QoS parameter $=y_{f, n} \vec{c}$

\subsection{Bandwidth Allocation}

For each fog device can denote and associate many IOT devices on the basis of SNR ratio. The transmission capacity is given by:

$X_{c, p}^{a}\left(x_{c, p}^{a}\right)=x_{c, p}^{a}\left(\log \left(1+\theta_{c, p}^{a}\right)\right)$

$x_{c, p}^{a}=$ allocated bandwidth for IOT and $x_{\max }^{a}=\sum_{a \in A} x_{c, p}^{a}$ denotes the maximum bandwidth, where

$\theta_{c, p}^{a}=\frac{h_{c, p}^{a}+A_{c, p}^{a}}{\alpha^{2}+j_{c, p}}$ denotes SNR ratio

When the fog devices again allocate sub-channel A to IOT devices $C ; h_{c, p}^{a}$ denotes transmission power. $A_{c, p}^{a}$ denotes the gain in the channel between the fog devices and $\alpha^{2}$ denotes the variance.

\subsection{Job Delay}

The job delay consists of queuing delay and the transmission delay. For each fog device $p \in P$ such that it requests for the job to many IOT devices. There is a process of data transmission that takes place.

(Mean traffic $=i_{p}$ (Packets /second), Packet Transmission Rate $=\rho_{p}$ and $O_{\text {avg }}=$ Average Mean Size). The average job delay denotes:

$\vee_{c, p}=\frac{O_{a v g}}{X_{c, p}^{a}\left(x_{c, p}^{a}\right)}+\frac{i_{p}}{i_{p}-\rho_{p}}$

\subsection{Bit-Error Rate Computation}

When data is transmitted to the interfacing IOT device and fog devices, there could be errors and corruption.

$\frac{X_{c, p}^{a}}{X_{c, p}^{a}}$ denotes the performance modulation

$z_{c, p}=\left\{\begin{array}{c}0.2 \times e^{\frac{-1.6 \times h_{c, p}^{a}}{\log l-1}} \\ 1\end{array}\right.$ if $h_{c, p}^{a} /\left(\alpha+j_{c, p}\right) \geq P_{p}$

$\mathrm{l}=$ Modulation Index

$h_{c, p}^{a} /(\alpha)=$ Per bit Energy to noise density, and

$t_{r}=$ Threshold for modulation 


\subsection{QoS Benefit Function}

The function illustrates to what extent QoS services have been achieved since this function incorporates all the parameters as weights defining job delay, bit error etc. that retard the rate of achieving QoS benefits.

$S_{c, p}^{a}\left(x_{c, p}^{a}, y_{f, n}^{\rightarrow c}\right)=\frac{y_{f, m 1}^{c} * X_{c, p}^{a}\left(x_{c, p}^{a}\right) * y_{f, m 2}^{c} \cdot\left(1-z_{c, p}\right)}{y_{f, m 3}^{c} * \theta_{c, p}^{a}}$

denotes QoS benefit function. Where $y_{f, m 1}^{c}, y_{f, m 2}^{c}, y_{f, m 3}^{c}$ are corresponding weights of throughput bit error rate and job delay.

\section{Problem Formulation}

The target of resource allocation in the network is to provide the maximum benefit of joint clubbing and resource allocation with QoS parameters in IOT.

Maximize $\tau_{c, p}, u_{c, p} \iiint \tau_{c, p} u_{c, p} S_{c, p}^{a} d p d c d a$

Where $d p, d c$, and $d a$ are the differential parameters w.r.t. p, c, a ( $\varepsilon \mathrm{P}, \mathrm{C}$, A respectively) and p, $\mathrm{c}$, a $\varepsilon \mathrm{P}, \mathrm{C}$, A respectively. The target function is being maximized for the distinct IOT device to services.

$\iint \tau_{c, p} u_{c, p} x_{c, p}^{a} \mathrm{dc} \mathrm{da}<=x_{\max }^{p}$

$\tau_{c, p} u_{c, p} X_{c, p}^{a}\left(x_{c, p}^{a}\right)>=\Omega$

Equations 6, 7, and 8 define the network resource allocation. $\Omega$ denotes minimum QoS requisites. $\tau_{c, p} u_{c, p}$ can be 1 if device is given a subchannel else 0 .

\section{Decision Parameter and Problem Solution}

The problem making function in equation, one would have been solved by knapsack problem 0/1 if there lies its order $O\left(2^{C * P * A}\right)$ which is NP hard and the condition prevails to be highly complex, so we try to solve the problem with SLP (systematic ladder process) as presented in Table 1 and try to evaluate the decision parameter which is done by the formation of RECK Algorithm. The various layers used in the Table 1 are also shown in Figure 1, where layer 3 consists of devices, layer 1 selects the IOT devices, and layer 2 is used for the performance of various algorithms in terms of throughput, bit error rate, and job delay.

Table1. Resource allocation scheme-Systematic Ladder Process (SLP)

\begin{tabular}{|l|l|}
\hline LAYER 1 & TARGET \\
\hline LAYER 2 & DECISION CRITERIA \\
\hline LAYER 3 & SUBSTITUE \\
\hline
\end{tabular}




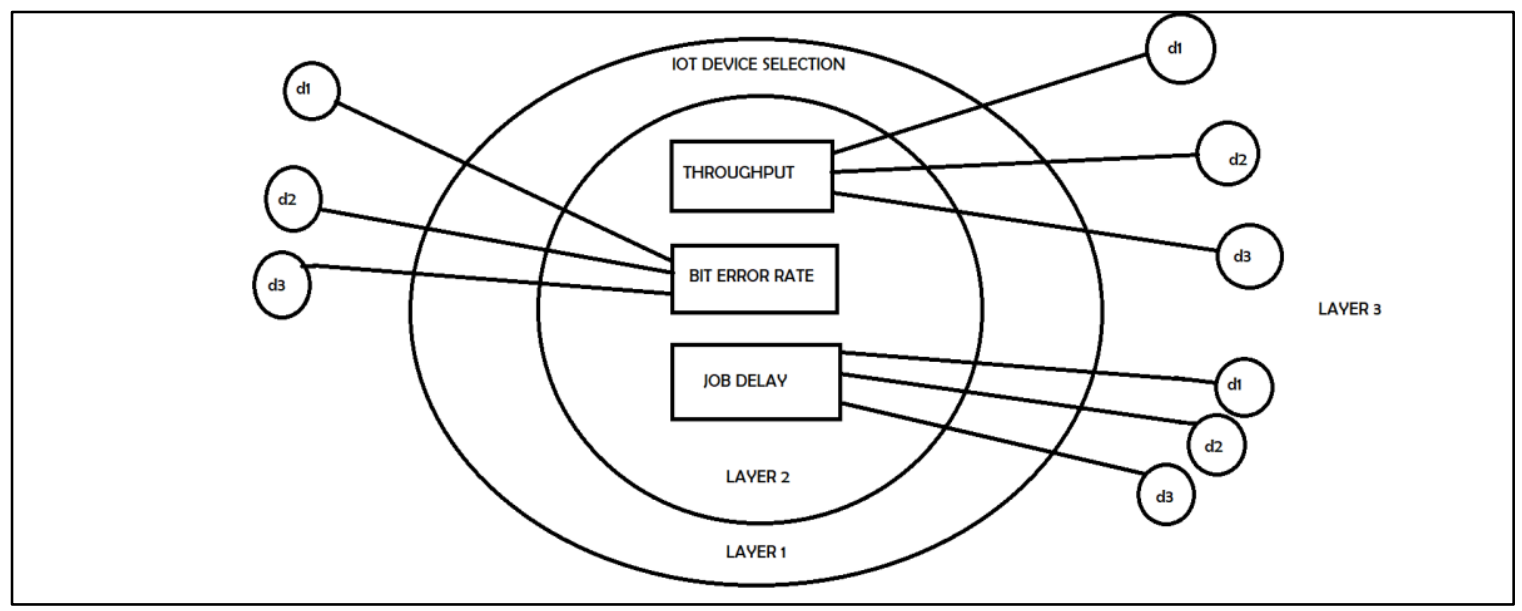

Figure 1. Resource allocation scheme over various layers

\section{Decision Parameter Evaluation RECK Algorithm}

Decision parameter is evaluated by the RECK algorithm (Proposed) given below:

1) Start

2) Let there are three cases Case 1, Case 2, Case 3 denoting device allocation by throughput, Bit error rate, job delay.

3) Priority being assigned in the fashion of order of Case 1, Case 2, Case 3 because job delay would be tolerated to an extent provided if there is minimised error.

4) Allocate the same normalised weight Wa to all devices say d1, d2, d3

5) Calculate the throughput, bit error rate and job delay for each device say $\mathrm{d} 1, \mathrm{~d} 2, \mathrm{~d} 3$.

6) Case 1: For Each device: Throughput $>$ (Job delay, Bit Error rate)

Preference of allocation will be according to throughput as preference is allocated to case 1 first.

7) (Randomised) allocation of weights according to throughput to the devices $\mathrm{d} 1, \mathrm{~d} 2, \mathrm{~d} 3$ as w1, w2, w3

8) Calculate $\mathrm{f} 1=\mathrm{Wa} * \mathrm{w} 1, \mathrm{f} 2=\mathrm{Wa} * \mathrm{w} 2, \mathrm{f} 3=\mathrm{Wa}^{*} \mathrm{w} 3$, where $\mathrm{f} 1, \mathrm{f} 2, \mathrm{f} 3$ are global decision parameter.

9) Maximum (f1, f2, f3) will fetch with the selection as d1, d2, d3 respectively for f1, f2, f3 being maximum value.

10) Case 2: Throughput < (Job delay > Bit Error rate) this will be given second preference for allocation of devices as job delay could be tolerated as compared to the error rates.

11) Randomised weight allocation to devices according to job delay as $\mathrm{w} 4$, w5, w6 for $\mathrm{d} 1$, d2, d3 respectively.

12) Calculate $\mathrm{f} 4=\mathrm{Wa} * \mathrm{w} 4, \mathrm{f} 5=\mathrm{Wa}^{*} \mathrm{w} 5, \mathrm{f} 6=\mathrm{Wa}^{*} \mathrm{w} 6$, where $\mathrm{f} 4, \mathrm{f} 5$, f6 are global decision parameter.

13) Minimum (f4, f5, f6) will be given the allocation to devices d1, d2, d3 for f4, f5, f6 being minimized.

14) Case 3: Throughput $<$ (Job delay < Bit Error rate) this will be given third preference for allocation of devices as job delay could be tolerated as compared to the error rates.

15) Randomised weight allocation to devices according to bit error rate as w7, w8, w9 for d1, $\mathrm{d} 2, \mathrm{~d} 3$ respectively. 
16) Calculate $\mathrm{f} 7=\mathrm{Wa} * \mathrm{w} 7, \mathrm{f} 8=\mathrm{Wa}^{*} \mathrm{w} 8, \mathrm{f} 9=\mathrm{Wa} * \mathrm{w} 9$ Where $\mathrm{f} 7, \mathrm{f} 8, \mathrm{f} 9$ are global decision parameter.

17) Minimum (f7, f8, f9) will be given the allocation to devices $d 1, d 2$, d3 for $f 4$, f5, f6 being minimized.

18) END

\section{Simulations}

Former deferred algorithm was found to be less efficient than systematic ladder process of amalgamation with RECK algorithm. The comparison is brought out between the two algorithms in the prospectus of the average bit rate, average job delay, bandwidth utilization, and throughput. In the standard comparison process, we have initially used no of fog devices to be 10 and no of IOT devices to be 50 .

Table 2. Illustration of the data comparison on the basis of throughput

\begin{tabular}{|c|c|c|}
\hline & \multicolumn{2}{|c|}{ Cumulative Distribution } \\
\hline Average throughput & DA Algorithm & RECK Algorithm \\
\hline 10 & 0.09 & 0.03 \\
\hline 20 & 0.16 & 0.12 \\
\hline 30 & 0.28 & 0.23 \\
\hline 40 & 0.34 & 0.3 \\
\hline 50 & 0.51 & 0.45 \\
\hline
\end{tabular}

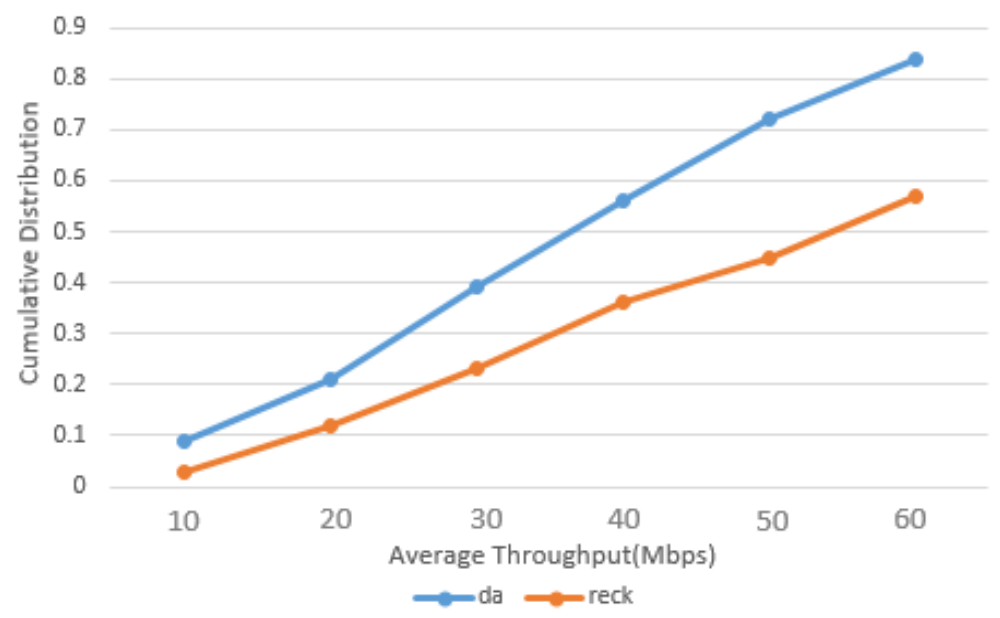

Figure 2. Comparison of DA and RECK algorithms on the basis of throughput

In the Figure 2, the comparison is based on the values of fog and IOT devices to be 10 and 50 respectively. The graph in Figure 2 clearly highlights in context of throughput RECK algorithm being much efficient, according to the data provided in various blocks of Table 2 . The cumulative distribution is being used for comparison. 
International Journal of Mathematical, Engineering and Management Sciences

Vol. 5, No. 6, 1312-1323, 2020

https://doi.org/10.33889/IJMEMS.2020.5.6.097

Table 3. Highlights the data of DA and RECK algorithm on the basis of average job delay

\begin{tabular}{|c|c|c|}
\hline & \multicolumn{2}{|c|}{ Commutative Distribution } \\
\hline Average job delay & DA Algorithm & RECK Algorithm \\
\hline .0001 & 0.09 & 0.03 \\
\hline 0.002 & 0.16 & 0.12 \\
\hline 0.003 & 0.28 & 0.23 \\
\hline 0.004 & 0.34 & 0.3 \\
\hline 0.005 & 0.51 & 0.45 \\
\hline
\end{tabular}

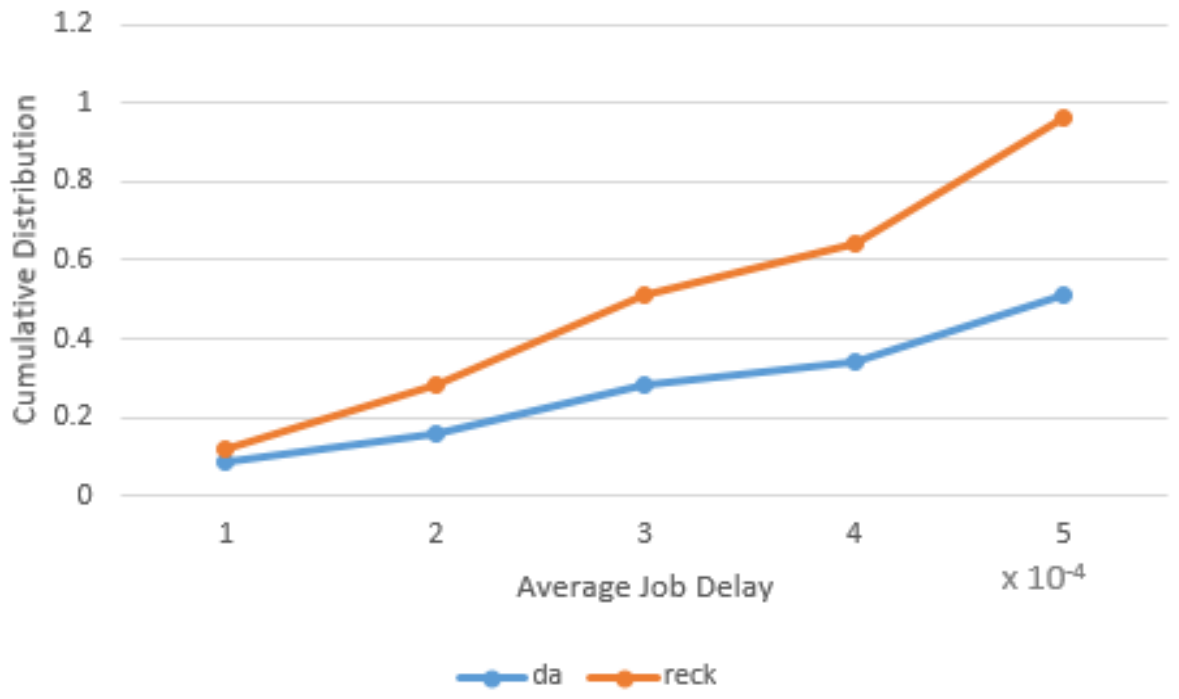

Figure 3. Comparative analysis of DA and RECK algorithm in the context of cumulative distribution and average job delay

Prioritizing the cases in the RECK algorithm clearly depicts the true picture of the comparison between the two algorithms on the context of Job delays. In Figure 3, the comparison is based on the values of fog and IOT devices to be 10 and 50 respectively. RECK algorithm turns out to be efficient in the Figure 3 on the basis of average job delay which implies RECK algorithm to be a better resource allocation algorithm than deferred algorithm. The comparison is seen between RECK \& DA on the basis of job delay in Figure 3 according to the data given in Table 3.

Table 4. Highlights the data of DA and RECK algorithm on the basis of average bit error rate

\begin{tabular}{|c|c|c|}
\hline & \multicolumn{2}{|c|}{ Cumulative Performance } \\
\hline Average bit Error rate & DA Algorithm & RECK Algorithm \\
\hline .001 & 0.03 & 0.09 \\
\hline .002 & 0.12 & 0.21 \\
\hline .003 & 0.23 & 0.39 \\
\hline .004 & 0.36 & 0.56 \\
\hline .005 & 0.45 & 0.72 \\
\hline
\end{tabular}




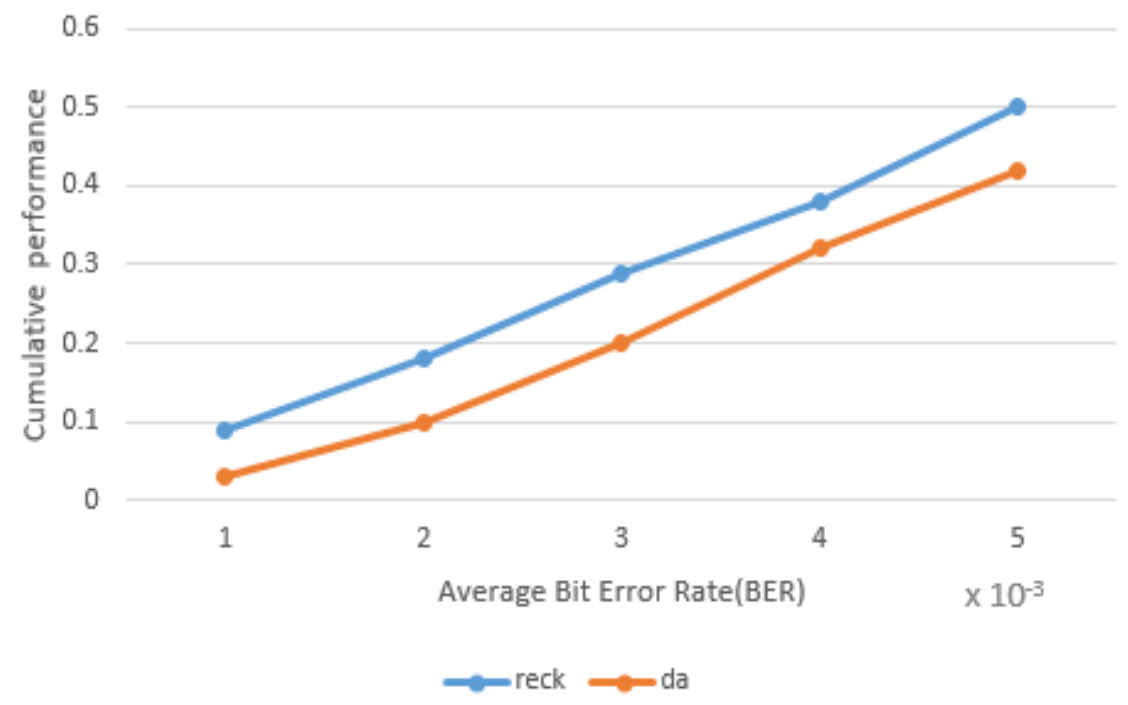

Figure 4. Comparative analysis of DA and RECK algorithm in the context of average bit error rate

In the Figure 4, the comparison is based on bit error rate and the values of fog and IOT devices are 10 and 50 respectively. In the context of RECK algorithm, according to the data evolved in Table 4 and the corresponding graph in Figure 4, it was clearly seen the error should be minimal and, in the figure, it is seen that the RECK algorithm proves to be efficient than DA.

Table 5. Highlights the data of DA and RECK algorithm in the context of average effectiveness

\begin{tabular}{|c|c|c|}
\hline & \multicolumn{2}{|c|}{ Average Effectiveness } \\
\hline No of IOT devices & DA Algorithm & RECK Algorithm \\
\hline 5 & 0.11 & 0.13 \\
\hline 15 & 0.14 & 0.16 \\
\hline 20 & 0.15 & 0.16 \\
\hline 25 & 0.13 & 0.18 \\
\hline 30 & 0.14 & 0.19 \\
\hline 40 & 0.16 & 0.18 \\
\hline 50 & 0.17 & 0.19 \\
\hline 60 & 0.12 & 0.17 \\
\hline
\end{tabular}

In the Figure 5, the comparison is based on average effectiveness and the values of fog and IOT devices are 10 and 50 respectively. The resource allocation algorithm should be effective for the proper working of the devices when being connected. By the data provided in Table 5 and its graph in Figure 5, between the algorithms the graph clearly shows that RECK is a better resource allocation algorithm than DA. 
International Journal of Mathematical, Engineering and Management Sciences

Vol. 5, No. 6, 1312-1323, 2020

https://doi.org/10.33889/IJMEMS.2020.5.6.097

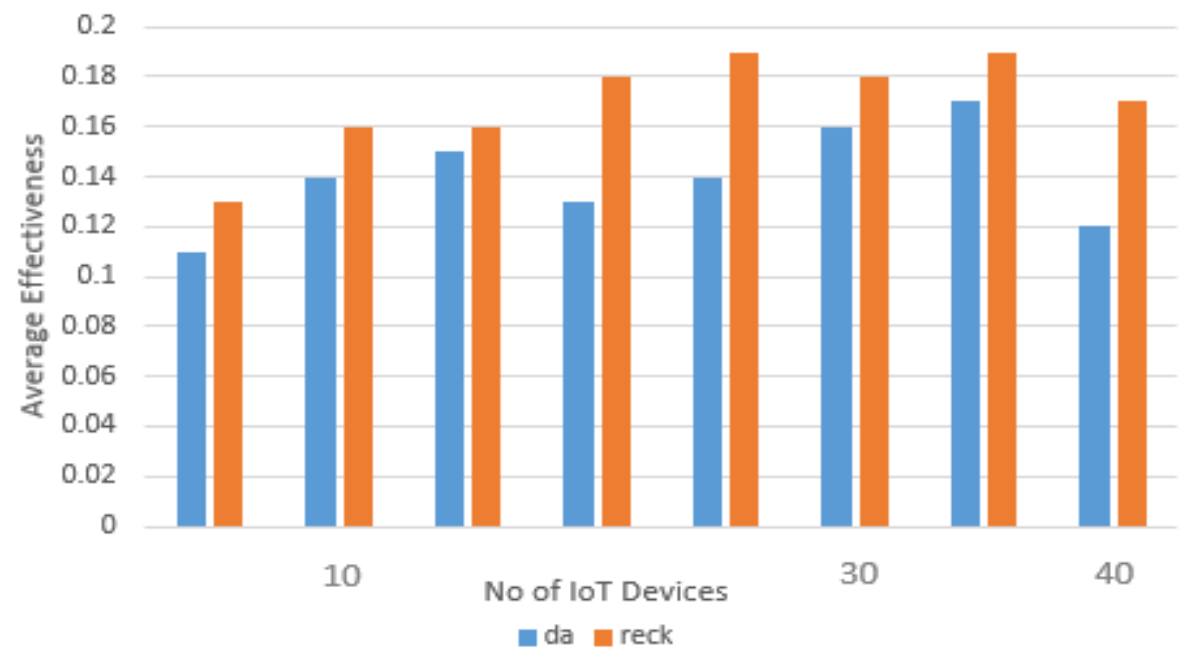

Figure 5. Comparative analysis of DA and RECK algorithm in the context of average effectiveness

\section{Conclusion}

In this paper, we have tried to draw attention to ensure the QoS quality of services for end-users by allocation of the resources being limited with efficiency to the heterogeneous applications associated with IOT. Thereby we tried to propose RECK algorithm approach for self-organizing distributed association of users and resource allocation that are better applicable and have scalability to the dense environment of fog computing. Unlike various schemes of resource allocation schemes for the applications associated with IOT with taking RECK algorithm into consideration in context with this algorithm analytics, resource demands, type of applications associated with QoS parameters. The simulations came out with a proposed approach inculcating the best resource allocation strategy and we compared the significant performance gains as compared to many resource allocation schemes available. The research carries a greater impact in creating the joint architecture of fog computing networks with better QoS, enhanced efficiency, low latency communication. It would be beneficial in the quick development of the internet and cloud technologies in our daily day to day life by reducing time in an IOT application in cloud \& fog computing with better performance. As the technology demands transformation one day the better algorithm would replace the concurrent algorithm with a better set of parameters, complexity and ideology.

\section{Conflict of Interest}

The authors confirm that the content of this article has no conflict of interest.

\section{Acknowledgments}

This research did not receive any specific grant from funding agencies in the public, commercial, or not-for-profit sectors. The authors sincerely appreciate the editor and reviewers for their time and valuable comments. 
International Journal of Mathematical, Engineering and Management Sciences

Vol. 5, No. 6, 1312-1323, 2020

https://doi.org/10.33889/IJMEMS.2020.5.6.097

\section{References}

Abedin, S.F., Alam, M.G.R., Kazmi, S.A, Tran, N.H., Niyato, D., \& Hong, C.S. (2019). Resource allocation for ultra-reliable and enhanced mobile broadband IoT applications in fog network. IEEE Transactions on Communications, 67(1), 489-502.

Bonomi, F., Milito, R., Natarajan, P., \& Zhu, J. (2014). Fog computing: a platform for internet of things and analytics. in Big Data and Internet of Tings: A Roadmap for Smart Environments, 546(1), 169-186.

Ding, Z., Liu, y., Choi, J., Sun, Q., Elkashlan, M., Chih-Lin, I., \& Poor, H.V. (2017). Application of nonorthogonal multiple access in LTE and 5G networks. IEEE Communications Magazine, 55(2), 185-191.

Fan, Q., \& Ansari, N. (2018). Towards traffic load balancing in drone-assisted communications for IoT. IEEE Internet of Things Journal 6(2), 3633-3640.

Gu, Y., Saad, W., Bennis, M., Debbah, M., \& Han, Z. (2015). Matching theory for future wireless networks: fundamentals and applications. IEEE Communication Journal, 53(5), 52-59.

Islam, S.M.R, Avazov, N., Dobre, O.A., \& Kwak, K.S. (2017). Power-domain non-orthogonal multiple access (NOMA) in 5G systems: potentials and challenges. IEEE Communications Surveys \& Tutorials, 19(2), 721-742.

Kiani, A., \& Ansari, N. (2018). Edge computing aware NOMA for 5g networks. IEEE Internet of Things Journal, 5(2), 1299-1306.

Li, P., Zhao, S., \& Zhang, R. (2010). A cluster analysis selection strategy for supersaturated designs. Computational Statistics \& Data Analysis. 54(6), 1605-1612.

Luo, E., Bhuiyan, M.Z.A., Wang, G., Rahman, M.A., Wu, J., \& Atiquzzaman, M. (2018). PrivacyProtector: Privacy-Protected patient data collection in IoT-based healthcare systems. IEEE Communications Magazine, 56(2), 163-168.

Peng, K., Lin, R., Huang, B., Zou, H., \& Yang, F. (2017). Link importance evaluation of data centre network based on maximum flow. Journal of Internet Technology, 18(1), 23-31.

Peng, M., Yan, S., Zhang, K., \& Wang, C. (2016). Fog-computing-based radio access networks: issues and challenges. IEEE Network, 30(4), 46-53.

Qiu, T., Zhang, Y., Qiao, D., Zhang, X., Wymore, M.L., \&. Sangaiah, A.K. (2018). A robust time synchronization scheme for industrial internet of things. IEEE Transactions on Industrial Informatics, 14(8), 3570-3580.

Rahman, M.A., Rashid, M.M., Hossain, M.S., Hassanain, E., Alhamid, M.F., \& Guizani, M. (2019). Blockchain and IoT-based cognitive edge framework for sharing economy services in a smart city. IEEE Access, 7, 18611-18621.

Sarkar, S., Chatterjee, S., \& Misra, S. (2015). Assessment of the suitability of fog computing in the context of internet of tings. IEEE Transactions on Cloud Computing, 6(1) 46-59.

Shirvanimoghaddam, M., Condoluci, M., Dohler, M., \& Johnson, S.J. (2017). On the fundamental limits of random non-orthogonal multiple access in cellular massive IoT. IEEE Journal on Selected Areas in Communications, 35(10), 2238-2252.

Song, L., Li, Y., Ding, Z., \& Poor, H.V. (2017). Resource management in non-orthogonal multiple access networks for $5 \mathrm{G}$ and beyond. IEEE Network, 31(4), 8-14.

Tian, G.L., Wang, M., \& Song, L. (2014). Variable selection in the high-dimensional continuous generalized linear model with current status data. Journal of Applied Statistics, 41(3), 467-483. 
International Journal of Mathematical, Engineering and Management Sciences

Vol. 5, No. 6, 1312-1323, 2020

https://doi.org/10.33889/IJMEMS.2020.5.6.097

Wang, S., Lei, T., Zhang, L., Hsu, C.H., \& Yang, F. (2016). Offloading mobile data traffic for QoS-aware service provision in vehicular cyber-physical systems. Future Generation Computer Systems, 61, 118127.

Yi, S., Li, C., \& Li, Q. (2015). A survey of fog computing: concepts, applications and issues. In Proceedings of the 2015 Workshop on Mobile Big Data (Mobidata '15), ACM, Hangzhou, China, 37-42.

Zhang, Y., Jiang, C., Wang, J., Han, Z., Yuan, J., \& Cao, J. (2018). Green Wi-Fi implementation and management in dense autonomous environments for smart cities, IEEE Transactions on Industrial Informatics, 14(4), 1552-1563. 